\title{
A FORMAÇÃO DOS CONCEITOS CIENTÍFICOS E O PROCESSO DE ENSINO- APRENDIZAGEM NOS ANOS INICIAIS DO ENSINO FUNDAMENTAL
}

\author{
Fernando Wolff Mendonça ${ }^{i} \odot 0000-0002-6193-4793$ \\ Universidade Estadual de Maringá - UEM \\ Maria Eduarda dos Santos ${ }^{\mathrm{ii}} \odot 0000-0001-9529-5750$ \\ Universidade Estadual de Maringá - UEM
}

RESUMO: Este artigo tem o escopo de analisar o processo de formação dos Conceitos Científicos, de acordo com os pressupostos da Pedagogia Histórico-Crítica e da Psicologia HistóricoCultural, sendo assim, buscou-se um aprofundamento teórico nos conceitos de pensamento, linguagem, funções psicológicas elementares, superiores e o processo de mediação. Este estudo classifica-se como de cunho bibliográfico e documental. Obtiveram-se os seguintes resultados por meio desta pesquisa, a formação dos conceitos é um ato real e complexo do pensamento que é influenciado pelos meios externos de desenvolvimento cultural (instrumentos e signos) e pelo processo de desenvolvimento das funções psíquicas. Condições como as experiências familiares, o ambiente escolar e professores mediadores podem influenciar no desenvolvimento dos conceitos científicos, verificou-se também a importância da união entre linguagem e pensamento para a formação dos conceitos científicos. Conclui-se que a interação social contribui para o processo de formação dos conceitos científicos e que os conceitos teóricos presentes na Teoria HistóricoCultural são importantes para elaboração de práticas pedagógicas contextualizadas e sistematizadas aos objetivos de aprendizagem dos alunos.

PALAVRAS-CHAVE: Conceitos Científicos. Pensamento. Teoria Histórico-Cultural.

\section{THE FORMATION OF SCIENTIFIC CONCEPTS AND THE TEACHING- LEARNING PROCESS IN THE EARLY YEARS OF FUNDAMENTAL EDUCATION}

\begin{abstract}
This article aims to analyze the formation process of Scientific Concepts, according to the assumptions of HistoricalCritical Pedagogy and Historical-Cultural Psychology, therefore, a theoretical deepening of the concepts of thought, language, psychological functions was sought. elementary, superiors and the mediation process. This study is classified as bibliographic and documentary. The following results were obtained through this research, the formation of concepts is a real and complex act of thought that is influenced by the external means of cultural development (instruments and signs) and by the process of development of psychic functions. Conditions such as family experiences,
\end{abstract}

the school environment and mediating teachers can influence the development of scientific concepts, there was also the importance of the union between language and thought for the formation of scientific concepts. We conclude that social interaction contributes to the process of forming scientific concepts and that the theoretical concepts present in the HistoricalCultural Theory are important for the development of contextualized and systematized pedagogical practices to students' learning objectives.

KEYWORDS: Scientific Concepts. Thought. Historical-Cultural Theory. 


\section{DOI 10.33872/revcontrad.v2nl.e015 | v. 2, n. 1 | Jan./Jun. 2021}

\section{Introdução}

Para o processo de estruturação desta análise selecionou-se como aporte teórico a Psicologia Histórico-Cultural (THC) e a Pedagogia Histórico-Crítica que possuem origem no materialismo histórico-dialético e advogam que o homem é um ser social e o seu desenvolvimento ocorre por meio da apropriação do conhecimento.

O homem é um animal racional que se diferencia dos demais animais por meio de sua inteligência, segundo a Psicologia Histórico-Cultural a capacidade do ser humano de desenvolver o psiquismo decorre das apropriações humano-genéricas disponibilizadas em sociedade, sendo assim a internalização desse conhecimento ocorre por meio das relações interpessoais que é referente ao conhecimento compartilhado entre pessoas e intrapessoais, que é resultado da soma das internalizações adquiridas pelos sujeitos, por meio do convívio em sociedade. Desse modo, Martins evidencia que "Os processos de internalização [...] instituemse baseados no universo de objetivações humanas disponibilizadas para cada indivíduo por meio da mediação de outros indivíduos, ou seja, processos educativos.” (MARTINS, 2016, p. 14).

A formação dos conceitos científicos é um ato real e complexo do pensamento, é somente por meio desses conceitos que o indivíduo adquire condições de reflexão e análise para atuar criticamente em sociedade, esses conceitos estabelecem uma série de relações e significados no pensamento dos indivíduos. O processo de formação dos conceitos científicos inicia-se por meio da união entre linguagem e pensamento que tem como consequência a expressão do conceito por meio da palavra.

Inicialmente, o pensamento restringe-se a questões afetivas e biológicas e representa uma produção interna do indivíduo. Já a linguagem é uma produção externa, sendo resultado de uma construção histórico-social que se organiza por meio de um sistema de símbolos e signos que são utilizados como meio de comunicação.

Nos primórdios do desenvolvimento, o pensamento infantil é permeado exclusivamente pelos conceitos espontâneos, entretanto, com o desenvolvimento da linguagem ocorre a complexificação do psiquismo por meio da aquisição de um sistema de transmissões de conceitos, sendo assim, a palavra representa a capacidade do indivíduo de transmitir um sistema de conceitos. Destarte que, esse é apenas o início do processo de formação dos conceitos científicos, pois esses conceitos caracterizam-se pela consciência, o controle e a sistematicidade 
e, além disso, pressupõe das funções psicológicas superiores, os conceitos científicos que, segundo Vygotsky (1998) apenas se desenvolvem na adolescência.

A escola é lócus privilegiado no processo de formação dos conceitos científicos, pois é por meio do ambiente escolar que a criança tem acesso ao conteúdo clássico e sistematizado, desse modo, é por meio do ambiente escolar que o indivíduo tem acesso as apropriações culturais mais complexas que são essenciais para a formação de um indivíduo crítico e emancipado, destarte que o indivíduo que não tem acesso ao conhecimento historicamente elaborado, não adquire condições de desenvolver os conceitos científicos e não consegue atuar criticamente em sociedade, pois não conhece efetivamente a realidade objetiva. Ou seja, conforme afirma Vygotsky: "O aprendizado adequadamente organizado resulta em desenvolvimento mental e põe em movimento vários processos de desenvolvimento que, de outra forma, seriam impossíveis de acontecer.” (VYGOTSKY, 1987, p. 101).

Em síntese, o presente artigo tem por objetivo investigar a formação dos conceitos científicos e compreender a importância dos conceitos da Teoria Histórico-Cultural e dos conhecimentos prévios do aluno na formação de conceitos, com o intuito de apontar possíveis melhoras no processo de ensino-aprendizagem. A metodologia selecionada para a análise no desenvolvimento desta pesquisa classifica-se como de cunho bibliográfico e documental, inicialmente foi realizado um levantamento bibliográfico a respeito do processo de formação dos conceitos científicos na Teoria Histórico-Cultural, assim buscou dialogar com os seguintes autores Lev Vygotsky, Leontiev, Lígia Martins e Marta Sueli de Faria Sforni. A pesquisa de documentos ocorreu por meio de um levantamento do índice de aprendizagem das crianças matriculadas no $3^{\circ}$ ano do Ensino Fundamental I, no Município de Terra Boa - PR.

\section{O papel da linguagem no processo de formação dos conceitos}

O homem caracteriza-se como um ser social que diferentemente dos demais animais é responsável por produzir signos e instrumentos culturais que correspondam às suas necessidades afetivas, educativas e biológicas. O desenvolvimento humano ocorre por meio da apropriação dos instrumentos de trabalho. $\mathrm{O}$ trabalho é um processo de humanização que ocorre conforme o homem apropria-se do conhecimento historicamente elaborado. Vale ressaltar que conforme afirma Saviani, "[...] o trabalho educativo é o ato de produzir, direta e intencionalmente, em cada indivíduo singular, a humanidade que é produzida histórica e coletivamente pelo conjunto dos homens" (SAVIANI, 2015, p. 287). Os instrumentos de 
trabalho classificam-se em: instrumentos físicos que se vinculam a atividade prática e instrumentos simbólicos que se relacionam ao desenvolvimento das funções mentais.

A linguagem é um sistema organizado de símbolos e signos que pode ser utilizado como meio de comunicação, sendo essencial no desenvolvimento do psiquismo. Pode-se ressaltar, que a espécie humana ao usufruir desse instrumento simbólico de comunicação, o modifica devido a sua capacidade de alterar os bens culturais conforme suas necessidades. O signo permite outra forma de representação dos objetos e fenômenos da realidade concreta, por meio do uso consciente da palavra, além disso, é um instrumento que auxilia na resolução de tarefas psicológicas, pode-se mencionar, nesse sentido, o auxílio da memória. Destaca-se que:

[...] a transformação das funções psíquicas elementares em funções psíquicas superiores revela-se um processo condicionado, e Vigotski (VIGOTSKY, 1997 apud MARTINS, 2016) identificou no signo o condicionante nuclear da requalificação do sistema psíquico humano (MARTINS, 2016, p.15).

A linguagem é um instrumento de auxílio na internalização de um sistema de símbolos e signos que compreende o significado das palavras e fornece meios para a abstração, generalização e internalização do conteúdo e isso desenvolve a consciência, característica essencial no processo de desenvolvimento dos conceitos científicos. Vale ressaltar que o uso consciente da palavra implica na compreensão mental do signo e do significado, assim afirma Mendonça (2017):

\begin{abstract}
A instrumentalidade simbólica altera ainda mais as formas de relações entre os homens, visto que, mediante gestos e som, modos rudimentares de comunicação, aperfeiçoados e transformados socialmente, surgem a linguagem humana, carregando mediante a palavra não apenas a dimensão sonora e visual, mas o instrumental simbólico pela significação; ou seja, o conceito existe em cada palavra (MENDONÇA, 2017, p. 49).
\end{abstract}

Esse instrumento simbólico é produto da ontogênese e resultado de uma construção histórico-social, sendo apropriada por meio das relações em sociedade. É um instrumento de suma importância no desenvolvimento humano, uma vez que fornece conceitos responsáveis por estruturar o pensamento e auxiliar no desenvolvimento de funções psíquicas complexas, como a atenção e a consciência.

Os processos psíquicos do homem sobre a terra se formam sob a influência do mundo dos objetos que o rodeiam, e daquelas pessoas com as quais determina uma relação; adquire dali uma linguagem que existe apenas de maneira objetiva, desde o princípio, como meio de comunicação e depois, também 
como veículo de conhecimento (SVIETKOVA, 1985, p. 21 apud MENDONÇA, 2017, p. 23).

Deste modo, a linguagem possibilita a aquisição de um conjunto de ideias e informações, e é por meio desse instrumento simbólico que o indivíduo internaliza o conteúdo das diferentes ciências e adquire o conhecimento historicamente elaborado, destaca-se que o professor deve buscar mediar às relações estabelecidas com a linguagem em sala de aula, para conduzir o aluno a um processo de análise e reflexão dos conteúdos apreendidos no ambiente escolar, uma vez que isso proporciona a formação de um sujeito crítico e reflexivo, que utiliza de modo consciente os conceitos.

\section{O processo de formação dos conceitos científicos}

Segundo a Teoria Histórico-Cultural, o homem é um ser social que, diferentemente dos animais, é responsável por produzir signos e instrumentos culturais que correspondam às suas necessidades biológicas, afetivas e culturais. Os signos são instrumento de qualificação do psiquismo, uma vez que proporcionam uma nova forma de representação da realidade concreta por meio da abstração e elaboração de uma imagem subjetiva da realidade objetiva.

A educação é uma prática social complexa que tem por objetivo promover a humanização dos sujeitos e a formação dos conceitos científicos, por meio da transmissão dos conhecimentos historicamente elaborados, é uma instituição social que tem a função de identificar e organizar os conhecimentos necessários e indispensáveis à formação e emancipação dos indivíduos.

[...] a atividade de ensino conquista uma natureza específica na forma de educação escolar, que desponta como um processo a quem compete oportunizar a apropriação dos conhecimentos historicamente sistematizados o enriquecimento do universo de significações-, tendo em vista a elevação para além das significações mais imediatas e aparentes disponibilizadas pela simples pertença cultural e pelas dimensões meramente empíricas dos fenômenos (MARTINS, 2016, p. 18).

A escola tem como função transmitir os conhecimentos clássicos, ou seja, é por meio da escola que o indivíduo tem acesso aos conteúdos de leitura, escrita, matemática e aos conhecimentos básicos de história e geografia. Esses conhecimentos são responsáveis por modificar a estrutura cognitiva dos sujeitos e por promover a ascensão das funções psicológicas elementares as funções psicológicas superiores e ao desenvolvimento dos conceitos científicos, 
destaca-se, também, que é somente por meio do conhecimento historicamente elaborado que o sujeito terá condições de atuar criticamente na sociedade.

O pensamento é resultado da atividade histórico-cultural dos indivíduos, uma vez que representa a capacidade do homem de dominar a natureza e fazer com que essa atenda aos seus propósitos, nos primeiros meses de vida o pensamento é um ato prático que assume características de origem primitiva e restringe-se a questões afetivas e biológicas, ou seja, a criança é incapaz de tornar-se consciente da atividade da mente, por exemplo, um bebê recémnascido ao sentir a necessidade do leite materno automaticamente chora e tal choro representa a atividade inconsciente da mente.

Conforme o homem adquire experiências histórico-sociais, o ato de pensar se desenvolve, e isso representa a complexificação do psiquismo. O processo de complexificação do psiquismo ocorre, primordialmente, no ambiente escolar por meio do pensamento em conceitos. Vygotsky afirma que:

O pensamento em conceitos é o meio mais adequado para conhecer a realidade porque penetra na essência interna dos objetos, já que a sua natureza não se revela na contemplação direta de um ou outro objeto isoladamente, [...]. O vínculo interno das coisas se descobre com a ajuda do pensamento por conceitos, já que elaborar um conceito sobre algum objeto significa descobrir uma série de nexos e relações do objeto dado com toda a realidade, significa incluí-lo no complexo sistema de fenômenos que o sustenta (VIGOTSKY, 1996, p. 79).

Os conceitos espontâneos são conceitos naturais do pensamento que se originam de modo inconsciente e involuntário, por meio das experiências cotidianas dos sujeitos.

O processo de formação dos conceitos científicos é um ato real e complexo do pensamento que se caracteriza por possuir um sistema hierárquico de inter-relações e pressupõe de determinado nível de desenvolvimento mental, pois a criança é incapaz de tornar-se consciente da atividade da mente, de ter o controle consciente dos conceitos, pois antes de tornar-se consciente dos conceitos é necessário que a criança tenha desenvolvido as funções psicológicas superiores. Vale ressaltar que os conceitos científicos emergem a partir de processos elementares que se caracterizam pelo comportamento inconsciente e pensamento não deliberado, como também que o desenvolvimento dos conceitos científicos não ocorre pelo mesmo processo dos conceitos cotidianos.

Os conceitos científicos são responsáveis por introduzir os conceitos espontâneos em relações mais amplas e abstratas, por meio de um processo de autorregulação que é influenciado por condições internas e externas no processo de desenvolvimento. Conforme Vygotsky: 
Os conceitos científicos, com suas atitudes totalmente distintas para o objeto, mediados através de outros conceitos com seu sistema hierárquico interno de relações mútuas, constituem a esfera em que a tomada de consciência dos conceitos, ou seja, sua generalização e domínio, surgem, ao que parece, em primeiro lugar. Uma vez que a nova estrutura da generalização surge em uma esfera do pensamento, se transfere depois, como qualquer estrutura [...] (VIGOTSKY, 1982, p. 213).

Os conceitos científicos desenvolvem-se no ambiente escolar de modo intencional, direcionado e sistematizado. Entretanto, ambos os conceitos são essenciais na aquisição das estruturas psicológicas que proporcionam o desenvolvimento intelectual infantil. Por exemplo, por meio da experiência e do contato com o meio social, a criança assimila o conceito de animal (peixe, gato, pássaro e jacaré). Conforme a criança é inserida no conhecimento sistematizado ocorre o desenvolvimento, complexificação e classificação desse conhecimento, ou seja, a criança pode aprender o conceito de animais terrestres, aquáticos, aéreos, carnívoros, onívoros etc.

Pode-se mencionar, também, como exemplo, o conceito de mãe. Esse conceito é adquirido por meio da experiência cotidiana, entretanto, ao adentrar ao ambiente escolar ocorre a complexificação desse conceito, isso quer dizer que a criança aprende a diferenciar esse conceito, aprende as diferenças entre o conceito de mãe afetiva e mãe biológica e as relações intrínsecas a esses termos como o processo de adoção e as relações de afetividade.

Além disso, conforme Martins, “O processo de desenvolvimento de conceitos, afirmou Vigotski, exige e articula-se a uma série de funções, a exemplo da percepção acurada, da atenção voluntária, da memória lógica, da comparação, generalização [...]” (MARTINS, 2016, p. 23). Sendo assim, o desenvolvimento dos conceitos científicos é extremamente dependente das funções psicológicas superiores.

As funções psicológicas elementares são as primeiras estruturas naturais e originais do pensamento dos indivíduos, essas reações pertencem ao complexo-dinâmico afetivo e biológico e caracterizam-se por reações automáticas, pelo comportamento inconsciente e pensamento não-reflexivo, sendo assim, nesse período, o comportamento do homem é similar ao dos animais irracionais, uma vez que o meio externo determina as reações dos sujeitos, a partir de uma relação estímulo-resposta. $\mathrm{O}$ indivíduo ao nascer possui apenas as funções psicológicas elementares conforme o mesmo adquire experiências histórico-sociais o ato de pensar se desenvolve representando uma forma de conduta complexa e superior. 
As formas superiores dos processos mentais possuem uma estrutura particularmente complexa; elas são delineadas durante a ontogênese. Inicialmente elas consistem em uma série expandida, completa, de movimentos manipulatórios que gradualmente se tornam condensados e adquirem o caráter de ações mentais (VIGOTSKI, 1956; 1960; GALPERIN, 1959).

Com o desenvolvimento das funções psicológicas superiores o homem adquire a capacidade de internalização de um sistema de símbolos e signos, de generalização e abstração do pensamento, pois essas funções caracterizam-se pela atenção, memória, comportamento consciente, pensamento abstrato, raciocínio dedutivo e capacidade de planejamento, promovem um processo de intelectualização do pensamento e complexificação do psiquismo, sendo assim, permitem ao indivíduo a aquisição de habilidades mais complexas de comunicação e, consequentemente, uma maior independência.

As funções psicológicas superiores tornam-se importante para o processo de formação dos conceitos científicos, pois estabelecem os mecanismos necessários para o surgimento da consciência e do controle. Salienta-se ainda que é impossível transmitir os conceitos científicos de modo estéril, pois para que ocorra o processo de desenvolvimento dos conceitos científicos é necessário que a criança se torne consciente dos próprios processos mentais, isso contribui significativamente para o processo de ensino-aprendizagem.

Sendo assim, a formação dos conceitos científicos vincula-se a uma série de questões, como as experiências cotidianas que influenciam no desenvolvimento das funções psicológicas elementares e estabelece relações com a formação das funções psicológicas superiores que são essenciais no processo de desenvolvimento dos conceitos científicos. Ressalta-se, assim, que a escola é a instituição social responsável por inserir a criança no conhecimento historicamente elaborado que é organizado e sistematizado de acordo com os objetivos de aprendizagem, desse modo, a escola é a principal responsável pelo processo de desenvolvimento dos conceitos científicos, pois "Na experiência cotidiana a criança centra-se no objeto e não tem a consciência de seus conceitos, já nos conceitos apreendidos na escola em junção com o adulto, esta consegue resolver melhor o uso consciente dos conceitos" (VYGOTSKI, 1998, p. 136).

Destaca-se que ao adquirir os conceitos científicos, a criança torna-se capaz de relacionar os conhecimentos aprendidos na experiência cotidiana com os conhecimentos historicamente elaborados, sendo assim, "Para se criar métodos eficientes para a instrução das crianças em idade escolar no conhecimento sistemático, é necessário entender o desenvolvimento dos conceitos científicos na mente da criança” (VYGOTSKY, 1998, p.103). 


\section{O papel do professor no processo de formação dos conceitos científicos}

A escola é responsável pela transmissão de um conjunto de saberes sistematizados e organizados de acordo com os objetivos de aprendizagem e o professor é o profissional responsável por conduzir esse processo de aquisição do conhecimento e transmissão dos conceitos. De acordo com Martins, tem-se que:

[...] a escolarização impõe-se como uma das condições decisivas para o desenvolvimento da capacidade de abstração, na ausência da qual os sujeitos permanecem reféns do sincretismo, sustentando suas ações pelas aparências dos fenômenos e não por aquilo que realmente são (MARTINS, 2016, p. 29).

Sendo assim, o indivíduo que não tem acesso a uma educação de qualidade não possui condições de atuar de modo crítico em sociedade, uma vez que o conhecimento historicamente elaborado permite que esse desenvolva a capacidade de abstração e de reflexão a respeito dos fenômenos, entretanto, sem o acesso ao conhecimento historicamente elaborado as ações dos indivíduos vinculam-se apenas a aparência externa dos fenômenos e esse não desenvolve a capacidade de análises mais amplas e profundas.

$\mathrm{O}$ ato de mediação direciona-se pela intencionalidade da ação e modificação da situação estimuladora. É por meio do ato de mediação que o contato humano com a realidade evolui do plano prático e sensorial em direção ao teórico e abstrato, pois é por meio do processo de mediação que o homem desenvolve o intelecto e evolui das funções psicológicas elementares em direção as funções psicológicas superiores, sendo assim, o ato de mediar durante o processo de ensino-aprendizagem escolar promove a ascensão e complexificação do psiquismo.

O processo de ensino-aprendizagem torna-se importante por ofertar aos alunos um processo mediado e direcionado de organização e sistematização dos conceitos. No entanto, destacamos que “[...] não é qualquer trabalho pedagógico que orienta o desenvolvimento das pessoas em direção de seu máximo desenvolvimento" (cf. MARTINS, 2016, p. 36).

O trabalho pedagógico deve estar adequadamente organizado e sistematizado aos objetivos de aprendizagem dos alunos e respaldado por meio dos conhecimentos científicos. Além disso, o trabalho pedagógico vincula-se essencialmente as ações do professor que é responsável por selecionar o conteúdo, os recursos didáticos e metodológicos empregados e por disponibilizar uma série de apropriações culturais aos indivíduos.

$\mathrm{O}$ ato de mediar não se constitui por meio da interação homem-objeto ou homem-meio, mas estrutura-se por meio de uma ação intencional e direcionada ao processo de aquisição do 
conhecimento. $\mathrm{O}$ ato de mediar é importante no processo de formação dos conceitos científicos à medida que o professor deve ser um mediador da aprendizagem dos alunos, este deve buscar estabelecer vínculos entre os conceitos espontâneos e os conceitos científicos com o objetivo de tornar o processo de ensino-aprendizagem real e significativo para o aluno.

O processo de formação dos conceitos científicos ocorre por meio da apropriação contínua do conhecimento e dos modos de comportamento do homem, assim evidencia-se a importância do professor e do processo de mediação. O professor deve ser um mediador da aprendizagem dos alunos de modo a identificar os conhecimentos já adquiridos pelas crianças, com o objetivo de se chegar as potencialidades do conhecimento, de forma a relacionar os conteúdos aprendidos na escola com as vivências externas da criança.

Vygotsky (1998) é responsável por estruturar o conceito de Zona de Desenvolvimento. A Zona de Desenvolvimento Real representa aquilo que o indivíduo já conhece e expressa a capacidade da criança de resolver problemas sem a ajuda de um adulto. Já a Zona de Desenvolvimento Proximal representa aquilo que o indivíduo pode conhecer por meio da ajuda de um adulto, ou seja, expressa a capacidade de solucionar problemas por meio da orientação de um adulto e, por fim, a Zona de Desenvolvimento Potencial representa tudo aquilo que o indivíduo tem a possibilidade de conhecer e aprender, mas ainda não conhece.

O professor deve se responsabilizar por identificar aquilo que o aluno conhece, para assim poder determinar, definir e conduzir o aluno a aquilo que este pode conhecer. Quanto maior a zona de desenvolvimento proximal da criança, maior é seu nível de desenvolvimento mental, pois Vygotsky afirma que "A experiência nos mostrou que a criança com a zona maior de desenvolvimento proximal terá um aproveitamento muito melhor na escola" (VYGOTSKY, 1998, p. 128).

Desse modo, a escola é responsável por transmitir uma série de saberes que promove a ascensão do psiquismo e o desenvolvimento dos conceitos científicos, sendo o professor, o profissional responsável por conduzir esse processo de aquisição do conhecimento e de formação dos conceitos científicos.

Entretanto, como mencionado anteriormente, as experiências cotidianas podem influenciar nesse processo, destaca-se ainda que o aprendizado escolar antecede $o$ desenvolvimento, pois esse deve ter por objetivo o de desenvolver um conjunto de funções e conceitos que a criança ainda não possui, ou seja, a aprendizagem escolar exerce um papel fundamental na aquisição dos conceitos científicos, uma vez que promove, na criança, uma percepção generalizante que é mediada desde o início por um conceito anterior e que colabora para que posteriormente a criança se torne consciente dos próprios processos mentais. 


\section{Resultados}

Este artigo apresenta a síntese dos estudos e pesquisas ocorridos durante o Projeto de Iniciação Científica (PIC), realizado no período de 01/11/2018 a 31/10/2018, sob orientação da Professora Doutora Maria Christine Berdusco Menezes. Também advém dos estudos e pesquisas realizados sob orientação do Professor Doutor Fernando Wolff Mendonça.

A metodologia selecionada para o desenvolvimento desta pesquisa classifica-se como de cunho bibliográfico e documental e o suporte teórico adotado foi a Teoria Histórico-Cultural. As leituras, estudos e sistematizações de obras e conceitos essenciais a esta pesquisa estão organizados em fichamentos e tabelas. A pesquisa de documentos ocorreu por meio de um levantamento dos índices de aprendizagem das crianças matriculadas no $3^{\circ}$ ano do Ensino Fundamental I do Município de Terra Boa - PR. Para tanto, selecionou-se para análise o Índice de Desenvolvimento da Educação Básica (IDEB).

Por meio de um levantamento observa-se os dados estatísticos do Instituto Nacional de Estudos e Pesquisas Educacionais Anísio Teixeira (INEP), a respeito do Índice de Desenvolvimento da Educação Básica (IDEB) das escolas do Ensino Fundamental, do Munícipio de Terra Boa - PR, é notável que as quatro escolas presentes na região apresentaram um IDEB positivo no ano de 2015, inclusive acima da meta prevista para o ano.

Quadro 1: Índice de Desenvolvimento da Educação Básica - IDEB - Município de Terra Boa - PR

\begin{tabular}{|l|l|l|}
\hline ESCOLA & IDEB & META \\
& OBSERVADO & PROJETADA \\
\hline Escola Municipal Professor Adriano Franco & 6.2 & 5.6 \\
\hline Escola Municipal Monteiro Lobato & 6.9 & 5.8 \\
\hline Escola Municipal Therezinha Aparecida Bagatin & 6.1 & 6.1 \\
\hline Escola Municipal Princesa Isabel & 6.6 & 6.4 \\
\hline
\end{tabular}

Fonte: INEP (2015).

Ao realizar a análise dos dados mencionados anteriormente deve-se ressaltar que as quatro escolas encontram-se situadas em um mesmo município e os recursos financeiros são distribuídos igualmente entre elas, no entanto, as escolas possuem diferentes Índices de Desenvolvimento na Educação Básica (IDEB), de acordo com os pressupostos da Teoria Histórico-Cultural (THC) e isso justifica-se devido ao processo de ensino-aprendizagem dos conceitos científicos ser influenciado por condições internas e externas ao processo de desenvolvimento, ou seja, o processo de formação dos conceitos científicos não vincula-se apenas a disponibilidade de recursos materiais disponibilizados para a apropriação aos 
indivíduos, como livros, revistas, laboratório de informática, salas de aulas adequadas ao processo de ensino-aprendizagem. Mas, relaciona-se primordialmente a qualidade das mediações ofertadas aos indivíduos durante o processo de ensino-aprendizagem, além disso, condições como a personalidade dos estudantes, o ambiente social, as relações familiares, a metodologia do professor, os conhecimentos prévios do aluno podem influenciar no processo de formação dos conceitos científicos.

Desse modo, verifica-se que esse estudo obteve os seguintes resultados: um amplo entendimento a respeito do processo de formação dos conceitos científicos que abrange um conjunto de conceitos como pensamento, linguagem, funções psicológicas superiores, funções psicológicas elementares e o processo de mediação. Assim, observa-se que o processo de formação dos conceitos científicos é influenciado por condições internas e externas no processo de desenvolvimento e o professor deve considerar os conhecimentos prévios do aluno para um processo de ensino-aprendizagem real e significativo.

Ressalta-se que a compreensão dos conceitos da Teoria Histórico-Cultural contribui para o processo de formação dos professores, pois uma formação teórica adequada possibilita práticas pedagógicas eficazes e contextualizadas ao processo de ensino-aprendizagem, de modo a proporcionar a criança formas de se estabelecer relações entre os conhecimentos que já possui e os conteúdos ensinados no ambiente escolar com o escopo de criar um conhecimento real e significativo para o aluno, que torne este sujeito crítico e emancipado para atuar em sociedade.

\section{Considerações Finais}

Conclui-se que a formação dos conceitos científicos é um ato real e complexo do pensamento que pressupõe determinado nível de desenvolvimento mental. Esses conceitos não podem ser ensinados de modo mecânico ou por meio de treinamentos, pois, apenas podem ser assimilados após certo nível de desenvolvimento mental, ou seja, no período denominado como primeira infância (0 a 3 anos) a criança ainda não é capaz de desenvolver os conceitos científicos, uma vez que ainda não desenvolveu as funções mentais necessárias para o desenvolvimento desses conceitos.

$\mathrm{Na}$ primeira infância a criança possui somente os conceitos espontâneos que se caracterizam como assistemáticos e involuntários, o início do desenvolvimento dos conceitos científicos ocorre quando a criança adentra ao ambiente escolar, pois, o conhecimento sistematizado e organizado de acordo com as necessidades de desenvolvimento dos estudantes 
reorganiza os conceitos espontâneos e inicia um processo de sistematização de conceitos. O ambiente escolar por meio da transmissão e mediação do conhecimento historicamente elaborado é responsável por modificar, ampliar e sistematizar os conhecimentos advindos das experiências cotidianas das crianças, sendo assim, os conceitos espontâneos e científicos influenciam-se por meio de um sistema hierárquico de inter-relações. Ou seja, o processo de formação dos conceitos científicos ocorre, primordialmente, no ambiente escolar, uma vez que nesse ambiente os sujeitos entram em contato com o conhecimento sistematizado. $\mathrm{O}$ ambiente cotidiano proporciona a aquisição dos conceitos espontâneos.

Para que ocorra um processo de ensino-aprendizagem real e significativo, o professor deve atuar como um mediador e construir práticas de ensino contextualizadas durante o processo de ensino-aprendizagem dos alunos e, além disso, esse profissional deve buscar considerar os conhecimentos advindos da experiência espontânea na formação dos conceitos científicos.

O professor mediador é responsável por transmitir o conteúdo de modo dialógico, isso significa que o professor não é o único detentor do conhecimento durante o processo de ensinoaprendizagem, mas os alunos também podem contribuir com suas opiniões e experiências, ou seja, a construção do conhecimento ocorre por meio das trocas entre professor-aluno, alunoprofessor e aluno-aluno.

O professor mediador é um elo fundamental na formação dos conceitos científicos, pois o mesmo é capaz de identificar as funções mentais já desenvolvidas pelos alunos e planejar atividades ou práticas de ensino para desenvolver as funções mentais ainda necessárias ao processo de formação dos conceitos científicos. Entretanto, para que isso aconteça é preciso que o professor tenha um embasamento teórico adequado e a compreensão do processo de formação dos conceitos científicos, sendo assim, o professor deve compreender os conceitos de pensamento, funções psicológicas elementares, funções psicológicas superiores, zona de desenvolvimento, conceitos científicos e conceitos espontâneos.

Sendo assim, evidencia-se a importância dos cursos de formação inicial e continuada de professores, uma vez que é por meio de uma formação inicial e continuada que oferte os elementos teóricos e práticos necessários a constituição da prática educativa do professor, que os professores terão condições de conhecer os conceitos teóricos da Teoria Histórico-Cultural e utiliza-la no ambiente escolar, de modo a agregar ao processo de ensino-aprendizagem dos estudantes.

Os pressupostos da Teoria Histórico-Cultural advogam que a escola é um espaço direcionado a humanização e emancipação dos sujeitos e que é a mediação do conhecimento 
historicamente elaborado que permite o desenvolvimento dos conceitos científicos e a formação de sujeitos críticos e emancipados para atuar em sociedade, sendo assim, existe dois elos de fundamental importância para a formação dos conceitos científicos, o conhecimento historicamente elaborado é somente por meio da aquisição deste conhecimento que o sujeito terá condições de identificar as relações de alienação presentes em sociedade e atuar de modo consciente e o professor que é o profissional responsável por transmitir esse conhecimento ao estudante, de modo dialógico, crítico e reflexivo.

Além disto, destaca-se que a interação da criança com os adultos e outras crianças favorece aprendizagens significativas que contribuem para o processo de formação dos conceitos científicos. Tal interação com o outro favorece o desenvolvimento do ato de pensar e a transformação das funções psicológicas inferiores em funções psicológicas superiores, que são as características básicas para o processo de desenvolvimento dos conceitos científicos.

\section{REFERÊNCIAS:}

ABRANTES, A.; FACCI, M.; MARTINS, L. Periodização Histórico-Cultural do Desenvolvimento Psíquico do nascimento a velhice. $1^{\text {a }}$ ed. Campinas-SP: Autores Associados, 2016.

FACCI, M. Vigotski e o processo ensino-aprendizagem: a formação de conceitos. In: MILLER, S.; MENDONÇA, S. Vigotski e a escola atual: fundamentos e implicações pedagógicas. Araraquara, SP: Junqueira \& Marin, 2006.

MARTINS, L. Desenvolvimento do pensamento e educação escolar: etapas de formação de conceitos à luz de Leontiev e Vigotski. Disponível em: <https://periodicos.ufsc.br/index.php/forum/article/view/1984-8412.2016v13n4p1572>.

Acesso em: 08 fev. 2018.

MENDONÇA, F. A linguagem escrita na trajetória formativa. In: MENDONÇA, F. A organização da atividade de ensino como processo formativo do professor alfabetizador: contribuições da Teoria Histórico-Cultural. Tese (Doutorado). Maringá: Biblioteca Central UEM, 2017.

SAVIANI; D. Pedagogia Histórico-crítica: Primeiras Aproximações. In: SAVIANI, D. Sobre a Natureza e Especificidade da Educação. 11. ed. Campinas, SP: Autores Associados, 2011.

SFORNI, M.; GALUCH, M. Aprendizagem conceitual nas séries iniciais do ensino fundamental. Curitiba: Editora UFPR, 2006.

VIGOTSKI, L. S. O desenvolvimento dos conceitos científicos na infância. In: VIGOTSKI, L. S. L. S. Vigotski Pensamento e Linguagem. 2. ed. São Paulo: Martins Fontes, 1998.

VIGOTSKY, L. S. A formação social da mente. São Paulo: Martins Fontes, 1998.

\footnotetext{
${ }^{\text {i }}$ Doutor em Educação (UEM). Professor adjunto do departamento de Pedagogia e do Programa de Pós-Graduação em Psicologia da UEM. Email: ferwmen@ hotmail.com

ii Acadêmica da graduação em Pedagogia - CRC-UEM. Email: mariaeduardasantos1999999@live.com
} 